\title{
Clinical consultations and investigations before and after discontinuation of endocrine therapy in women with primary breast cancer
}

\author{
Derrick Lopeza,f, Anna Kemp-Caseya ${ }^{a}$, Christobel Saunders ${ }^{b}$, Elizabeth \\ Roughead ${ }^{c}$, Frances Boyled, Max Bulsara a and David Preen ${ }^{a}$ \\ a Centre for Health Services Research, School of Population and Global Health, University of Western Australia, Perth \\ b School of Surgery, University of Western Australia, Perth \\ ' Quality Use of Medicines and Pharmacy Research Centre, School of Pharmacy and Medical Sciences, University of South Australia, Adelaide \\ a Patricia Ritchie Centre for Cancer Care and Research, Mater Hospital, University of Sydney, NSW, Australia \\ e Institute for Health Research, University of Notre Dame, Fremantle, WA, Australia \\ ${ }^{\dagger}$ Corresponding author: derrick.lopez@uwa.edu.au
}

\section{Article history}

\section{Publication date: July 2017}

Lopez D, Kemp-Casey A, Saunders C, Roughead E, Boyle F, Bulsara M, Preen D. Clinical consultations and investigations before and after discontinuation of endocrine therapy in women with primary breast cancer. Public Health Res Pract. 2017;27(3):e2731726. https://doi.org/10.17061/phrp2731726

\section{Key points}

- Previous research shows that $58 \%$ of Australian women using endocrine therapy for primary invasive breast cancer discontinued treatment before the recommended 5 years

- We find no evidence to suggest that early discontinuation of endocrine therapy is associated with recent clinical contact or investigations for cancer recurrence or metastasis

- Women who discontinue endocrine therapy early are less likely to consult their general practitioner or surgeon/oncologist in the 6 months following discontinuation than those who remained on therapy

\section{Abstract}

Objective: Although clinical trials recommend that women with hormonedependent primary breast cancer remain on endocrine therapy for at least 5 years, up to $60 \%$ discontinue treatment early. We determined whether these women had consulted with clinicians or had investigations for cancer recurrence or metastasis around the time they discontinued endocrine therapy, and whether clinical contact continued after discontinuation.

Methods: We performed case-control and cohort studies of women from the 45 and Up Study who were diagnosed with invasive primary breast cancer between January 2003 and December 2008, and who had $\geq 12$ months of anastrozole, exemestane, letrozole or tamoxifen subsequently dispensed.

Results: Women who consulted general practitioners and surgeons/oncologists, and women who had breast ultrasound/mammogram were just as likely to discontinue endocrine therapy within 30 days as those who did not consult these clinicians or have this investigation. In the 6 months after early discontinuation, women who discontinued endocrine therapy were less likely to consult general practitioners (adjusted risk ratio [RR adj] 0.80; 95\% confidence interval [Cl] 0.75 , $0.86)$ and surgeons/oncologists $\left(\mathrm{RR}_{\mathrm{adj}} 0.62 ; 95 \% \mathrm{Cl} 0.54,0.72\right)$ than those who remained on therapy.

Conclusions: For most women, endocrine therapy discontinuation did not appear to follow consultation with doctors managing their breast cancer treatment or investigations for recurrence or metastasis. However, women who discontinued endocrine therapy were less likely to consult their general practitioner or surgeon/oncologist in the 6 months following discontinuation than those who remained on therapy. Of the clinician groups studied, general practitioners are best placed to engage and support women to continue pharmacotherapy. However, mechanisms are needed to prompt clinicians to do this at every visit. 


\section{Introduction}

Endocrine therapy is an established adjuvant treatment for women with hormone-dependent primary breast cancer. ${ }^{1}$ Despite clinical trials showing that endocrine therapy halves the risk of recurrence and reduces cancer-related mortality when taken for at least 5 years ${ }^{2-6}$, many women discontinue treatment early ${ }^{7,8}$, leaving them at an increased risk of cancer recurrence and death. ${ }^{8,9}$ We have previously shown that $58 \%$ of Australian women using endocrine therapy for primary invasive breast cancer discontinued treatment before the recommended 5 years. ${ }^{10}$ We were interested to determine if these women consulted with clinicians or had investigations for cancer recurrence or metastasis performed at or around the time the decision to discontinue endocrine therapy was made. This may reflect clinical consultation and decision making relating to issues such as side-effect management or continuing benefit of pharmacotherapy. In addition, we examined whether clinical follow-up continued after endocrine therapy discontinuation.

We hypothesised three possible relationships between discontinuation of endocrine therapy and clinical contact or investigations performed. Firstly, discontinuation may follow consultation with doctors managing the women's breast cancer treatment or side-effects of endocrine therapy (e.g. musculoskeletal pain). Secondly, discontinuation may follow negative test results (i.e. 'good news') from screening for recurrence or metastasis. Thirdly, discontinuation may co-occur with loss of clinical follow-up after discontinuing therapy. Understanding this relationship is important to design appropriate interventions to support women to continue endocrine therapy for the recommended period. For example, if discontinuation is associated with negative screening test results for either local or distant disease, then an appropriate intervention, such as encouragement and reinforcement of the benefits of continuing endocrine therapy, could be provided by the clinician when patients receive this news.

If clinical consultation and investigations are not related to discontinuation, this may suggest that clinicians are unaware of a woman's endocrine therapy status and that every clinical contact is an important opportunity to re-emphasise the importance of maintaining endocrine therapy and support patients to maintain use. Accordingly, the aim of this study was to determine whether women receiving endocrine therapy for primary breast cancer had an outpatient clinician visit or investigation for cancer recurrence and metastasis in the weeks before endocrine therapy discontinuation, and if clinical follow-up occurred after discontinuation.

\section{Methods}

We conducted case-control and cohort studies using administrative linked data and survey data of women from the 45 and Up Study who commenced endocrine therapy for primary breast cancer.

\section{Primary study base}

Participants were drawn from the Sax Institute's 45 and Up Study, a cohort of about 267000 adults (143 014 women) in New South Wales (NSW), Australia, aged $\geq 45$ years. ${ }^{11}$ Participants joined the study between January 2006 and April 2009, and completed a detailed baseline questionnaire of demographic, behavioural and healthrelated items. All participants recruited to the 45 and Up Study also provided written informed consent to have their health claims data that are routinely collected by the Australian Government Department of Human Services linked, and for these data to be provided to third-party researchers for approved projects.

\section{Data sources and linkage}

We accessed unit-record, linked data from: 1) the 45 and Up Study baseline survey; 2) NSW Admitted Patient Data Collection (hospital data); 3) NSW Cancer Registry; 4) Medicare Benefits Schedule (MBS) claims; 5) Pharmaceutical Benefits Scheme (PBS) claims; and 6) NSW Registry of Births Deaths \& Marriages. PBS and MBS data were supplied by the Department of Human Services and deterministically linked to the 45 and Up Study baseline data. The remaining datasets were probabilistically linked by the Centre for Health Record Linkage ${ }^{12}$, with quality audits showing fewer than $0.5 \%$ false-positive links. ${ }^{13}$ The study period was from 1 January 2003 to 30 November 2011.

\section{Study participants}

Participants drawn from the primary study base for this study were women with a diagnosis of invasive primary breast cancer on the NSW Cancer Registry between January 2003 and December 2008, and who had $\geq 12$ months of PBS-subsidised anastrozole, exemestane, letrozole or tamoxifen subsequently dispensed. Furthermore, to allow a 1-year follow-up after discontinuation, women were excluded if they discontinued endocrine therapy after 31 October 2010. Although there was increasing use of an aromatase inhibitor at some point in the treatment during this time, overall there was no change in the recommendation to use endocrine therapy in the study period.

\section{Selection of cases and controls}

Cases were defined as women who had commenced endocrine therapy after diagnosis and subsequently had no dispensing of endocrine therapy for a period greater than 180 days ('discontinuation') before the end of followup (cancer recurrence, death, end of study period, or 4 years of therapy - whichever occurred first). ${ }^{10}$ Women who switched endocrine therapy treatment (e.g. tamoxifen to letrozole) before the end of follow-up were not classified as cases. The date of discontinuation was the last recorded dispensing date plus the supply period..$^{10}$ We excluded women who discontinued endocrine therapy after 4 years 
because clinical concern would be greatest for those who stopped before this time and discontinuation after 4 years may have been planned.

Each case was randomly assigned to one control (defined as women with primary breast cancer who did not discontinue endocrine therapy during follow-up). Because each woman had a different endocrine therapy initiation date, and to ensure comparable follow-up periods, we assigned pseudo-discontinuation dates to each control based on the duration on endocrine therapy of their randomly assigned case. ${ }^{14}$

Given the inclusion criteria described, we identified 261 cases, and sufficient cases and controls for a 1:1 match.

\section{Ascertainment of side-effects, recurrence and death}

Ascertainment of endocrine therapy side-effects, recurrence and death in this cohort has been described in detail previously. ${ }^{10}$ In brief, dispensing of medicines used to treat anxiety, depression, hot flushes, musculoskeletal pain, osteoporosis and vaginal atrophy observed only after initiation of endocrine therapy were used to identify newonset side-effects. Breast cancer recurrence was identified from: 1) specified surgeries (lumpectomy, mastectomy, oophorectomy), chemotherapy and radiotherapy occurring for the first time $>18$ months from the date of diagnosis or $>12$ months after previous claims for these events; or 2) first dispensing of medicines (capecitabine, lapatinib, medroxyprogesterone, megestrol, toremifene, vinorelbine) indicated only for advanced breast cancer. Date of death was ascertained from the NSW Registry of Births Deaths \& Marriages.

\section{Ascertainment of clinical consultations and investigations}

Specified clinical consultations and investigations were selected after expert clinical consultation to derive the most appropriate services to analyse to test our hypothesised relationships. Clinical contacts of interest included consultations with general practitioners and outpatient consultations with oncologists, surgeons, rheumatologists or gynaecologists during the 30 days before discontinuation of endocrine therapy (Supplementary Table 1, available from: researchdataonline.research.uwa.edu.au/ handle/123456789/3001). These were ascertained from the MBS data.

Investigations related to screening for cancer recurrence (namely mammogram or breast ultrasound) and metastasis (isotope bone scan or fluorodeoxyglucose positron emission tomography [FDG-PET] or computed tomography [CT] scan) performed up to 30 days before discontinuation of endocrine therapy were also ascertained from the MBS data (Supplementary Table 2, available from: researchdataonline.research.uwa.edu. au/handle/123456789/3001). All post-breast cancer mammograms in NSW are done with Medicare rebates and recorded in the MBS data. To determine whether women had clinical follow-up after discontinuation, we followed them for 6 months after discontinuation. Most prescriptions provide a supply for up to 6 months, and an earlier study showed that most breast cancer patients attend consultations every 6 months. ${ }^{15}$

\section{Other covariates}

Residential location, annual household income, highest level of education and country of birth were reported by participants at recruitment to the 45 and Up Study. Residential location was coded using the Accessibility/ Remoteness Index of Australia. ${ }^{16}$ Cancer stage was determined from the NSW Cancer Registry. We calculated the number comorbidities using the Australian modification of Rx-Risk-V, a prescription-based comorbidity index, and using person-level data from PBS claims for 12 months before discontinuing endocrine therapy. ${ }^{17,18}$ We excluded malignancy from the comorbidity count because all women had a history of malignancy. Concession-card status was determined because the PBS dataset did not capture dispensing to general beneficiaries (i.e. nonconcession cardholders) when the medicines cost less than the copayment. ${ }^{19}$

\section{Statistical analysis}

We initially used t-tests and chi-square tests to compare continuous and categorical characteristics, respectively (demographic and clinical), of cases and controls. For the analyses of previous clinical contacts and investigations for cancer recurrence on endocrine therapy discontinuation, a case-control study design was used. For these, multivariate conditional logistic regression analyses were used to determine the adjusted odds ratio $\left(\mathrm{OR}_{\mathrm{adj}}\right)$ of discontinuing endocrine therapy within 30 days following exposure to specified clinical contacts and investigations for cancer recurrence.

For analyses on clinical contacts following endocrine therapy discontinuation, a cohort study design was used. For these, multivariate Poisson regression analyses were used to determine the likelihood of cases having clinical contact in the 6 months following discontinuation compared with controls. With binary outcomes, the exponentiated coefficients from the multivariate Poisson regression represent adjusted risk ratios $\left(R R_{a d i}\right)$ rather than incidence rate ratios. ${ }^{20,21}$ We used risk ratios because odds ratios are biased estimators of risk when the prevalence is high for the outcome being investigated (e.g. general practitioner consults).

All models were controlled for a range of covariates, including age at endocrine therapy discontinuation, highest level of education, annual household income, area of residence, country of birth, stage, number of comorbidities, concession-card status, family history of breast cancer and new-onset side-effects. Because women with newonset side-effects could be more likely to consult a clinician 
to manage these problems, we included the interaction term "discontinued endocrine therapy*new-onset side effect" in regression analyses for clinical consults to determine if there was any effect modification. We also assessed the interaction term "discontinued endocrine therapy ${ }^{\star}$ concession card" as the likelihood of clinical consultations may differ between those on concession and general beneficiaries. Aggregated counts of less than five were masked (presented as $<5$ ) to protect patient confidentiality. Analyses were performed using Stata.

\section{Ethics statement}

This study was conducted in accordance with Australian law. The consent procedure for entry to the 45 and Up Study was approved by the University of NSW Human Research Ethics Committee and the Australian Government Department of Health. The current study also received approval from the University of Western Australia Human Research Ethics Committee (approval RA/4/1/4589), and the NSW Population and Health Services Research Ethics Committee (approval HREC/11/CIPHS/35).

\section{Results}

\section{Demographic and clinical characteristics of cases and controls}

Of the 1531 women who commenced endocrine therapy, we identified 261 cases who discontinued endocrine therapy after $1-4$ years (median duration 1.8 years) of therapy. Cases and controls were similar in age, highest level of education attained, annual household income, area of residence, concession-card status, number of comorbidities, family history of breast cancer and new-onset side-effects (Supplementary Table 3, available from: researchdataonline.research.uwa.edu.au/ handle/123456789/3001). There were marginally more Australian-born women in the control than the case group (77.0\% vs $71.6 \%, p=0.037)$. Fewer than 10 women $(<3.8 \%)$ in the case and control groups were lost to follow-up because of death in the year following endocrine therapy discontinuation. In the control group, similar numbers of women had localised and regionalised cancers (47.5\% vs $48.7 \%$, respectively), whereas in the cases there were more women with localised than regionalised cancers (62.8\% vs $33.0 \%$, respectively).

\section{Endocrine therapy discontinuation within 30 days of specified clinical consultations}

Similar numbers of cases and controls consulted with general practitioners (43.7\% vs $44.8 \%, p=0.791)$, surgeons/oncologists (16.9\% vs $14.2 \%, p=0.397)$ and rheumatologists/gynaecologists (3.1\% vs $<1.9 \%, p=0.243$ ) in the 30 days before endocrine therapy discontinuation (Supplementary Table 4, available from: researchdataonline. research.uwa.edu.au/handle/123456789/3001). In the fully adjusted regression models, women who consulted general practitioners $\left(\mathrm{OR}_{\mathrm{adj}} 0.91 ; 95 \% \mathrm{Cl} 0.62,1.33\right)$ and surgeons/oncologists $\left(\mathrm{OR}_{\text {adj }} 1.44 ; 95 \% \mathrm{Cl} 0.87,2.41\right)$ had similar odds of discontinuation within 30 days as those who did not consult these clinicians. Women who consulted rheumatologists/gynaecologists were 3.03 times $(95 \% \mathrm{Cl}$ $0.72,12.69)$ more likely to discontinue within 30 days than those who did not consult this type of specialist, but they represent $<5 \%$ of women and this was not statistically significant. Adjusted odds ratios for general practitioner and surgeon/oncologist consults were similar to their unadjusted estimates.

\section{Endocrine therapy discontinuation within 30 days of specified clinical investigations}

Similar numbers of cases and controls discontinued endocrine therapy within 30 days of having a breast ultrasound/mammogram (6.5\% vs $8.4 \%, p=0.405$ ). However, twice the number of cases had bone study/ FDG-PET/CT scan than controls (5.7\% vs $2.7 \%, p=0.081$ ) (Supplementary Table 5, available from: researchdataonline. research.uwa.edu.au/handle/123456789/3001). In the multivariate analyses, there were no significant differences in the odds of discontinuing endocrine therapy within 30 days in women who had a breast ultrasound/ mammogram $\left(\mathrm{OR}_{\mathrm{adj}} 0.73 ; 95 \% \mathrm{Cl} 0.37,1.44\right)$ and bone

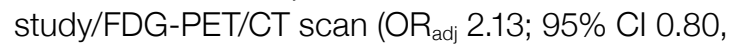
5.70) compared with women who did not have these investigations. Adjusted odds ratios for these clinical investigations were similar to their unadjusted estimates.

\section{Consultations in the 6 months following endocrine therapy discontinuation}

In the 6 months following endocrine therapy discontinuation, fewer cases than controls had consulted with general practitioners $(79.3 \%$ vs $97.3 \%, p<0.001)$ and surgeons/oncologists (48.3\% vs $78.9 \%, p<0.001$ ) (Supplementary Table 6, available from: researchdataonline. research.uwa.edu.au/handle/123456789/3001). Similar but small numbers had consulted a rheumatologist or gynaecologist $(9.2 \%$ vs $8.8 \%, p=0.878)$. In the multivariate model, women who discontinued endocrine therapy were less likely to consult general practitioners $\left(\mathrm{RR}_{\mathrm{adj}} 0.80 ; 95 \%\right.$ $\mathrm{Cl} 0.75,0.86)$ and surgeons/oncologists $\left(\mathrm{RR}_{\mathrm{adj}} 0.62 ; 95 \% \mathrm{Cl}\right.$ $0.54,0.72)$. The interaction terms "discontinuing endocrine therapy*new-onset side effects" and "discontinuing endocrine therapy ${ }^{\star}$ concession card" were not significant in the regression analyses for outcomes relating to general practitioner, surgeon/oncologist and rheumatologist/ gynaecologist consults.

\section{Discussion}

We were interested to determine whether women with a diagnosis of primary breast cancer who discontinued endocrine therapy within 4 years of treatment initiation 
had consulted clinicians, or had investigations for cancer recurrence or metastasis at or around the time they discontinued pharmacotherapy. Such information would help to contextualise the high levels of early endocrine therapy discontinuation that have been reported ${ }^{7,8,22-25}$ in terms of clinical contact and investigations performed. We found that women who consulted general practitioners and surgeons/oncologists, and women who had breast ultrasound/mammogram were just as likely to discontinue endocrine therapy within 30 days as those who did not consult these clinicians or have this investigation. Women who discontinued endocrine therapy were less likely to consult general practitioners and surgeons/oncologists in the 6 months following discontinuation.

We did not find any evidence to suggest that women discontinued endocrine therapy following recent clinical contact or investigations related to recurrence or metastasis. Encouragingly, many women continued to consult clinicians (especially general practitioners) after early discontinuation, although they were less likely to do so than those who continued endocrine therapy. This perhaps emphasises the need for every clinical contact, including those where investigation results are discussed, to be used as an opportunity for discussion with the patient about the importance of ongoing endocrine therapy for at least the first 5 years (as is recommended in current clinical guidelines ${ }^{2-6}$ ) or possibly longer (more recent data supports endocrine therapy use for 10 years ${ }^{26}$ ). Importantly, a recent qualitative study of breast cancer patients in the UK found that healthcare professionals did not routinely or systematically monitor the patient's adherence to endocrine therapy, and few women reported having the opportunity to discuss side-effects or the potential options available with their clinician. ${ }^{27}$ Kostev et al. suggest that, for women to remain on endocrine therapy, clinicians need to clearly communicate the seriousness of the condition, the importance of the treatment and its potential side-effects, and motivate the patient to take the medicine as prescribed. ${ }^{28}$

Of the three groups of clinicians investigated here, general practitioners are arguably best placed to encourage and support women with their endocrine therapy. Most (79\%) women who discontinued endocrine therapy had consulted their general practitioner in the 6 months following discontinuation, which was considerably higher than seen for other clinician types. The role of the general practitioner is even more important in rural and remote areas because women living in these areas have limited access to cancer specialists. ${ }^{29}$ General practitioner contact is likely to be more regular and frequent than specialist consultations, and therefore provides more opportunity to support endocrine therapy. On the other hand, there may be other competing issues for care (e.g. management of other chronic conditions) when women contact their general practitioner. Given time constraints, the general practitioner may be likely to focus on the condition related to the patient's reason for contact and may overlook the ongoing breast cancer pharmacotherapy regime, especially if it has been a number of years since the initial treatment. There needs to be mechanisms in general practice to prompt the clinician to engage and motivate these women to continue pharmacotherapy at every visit, and to contact women who have not had a recent consult. Consideration could also be given to engaging pharmacists in activity to support persistence with therapy, as all repeat dispensing for endocrine therapy is provided by the pharmacy.

In advocating for more clinician engagement and motivation, we are assuming that the decision to discontinue endocrine therapy was made solely by the patient. However, one study using self-reported data showed that the decision to discontinue endocrine therapy early was made with the clinician in $65 \%$ to $74 \%$ of women who discontinued. ${ }^{30}$ It is possible there was underreporting of self-initiated discontinuation, underascertainment of cancer recurrence (as a reason for discontinuation), or that women may have misunderstood the discussion they had with their doctor given the self-reported information used. A qualitative study of 30 women found that three women had discontinued endocrine therapy based on the decision of their clinician as they were considered to be low risk for cancer recurrence. ${ }^{27}$ However, this study did not indicate if the women stopped because they were nearing 5 years since treatment initiation.

\section{Strengths and limitations}

To our knowledge, this is the first study to examine clinical consultations and investigations before and after discontinuing endocrine therapy. We used administrative medical records for a heterogeneous population-based community sample for which all publicly subsidised endocrine therapy, outpatient consultations and clinical investigations were captured. We did not have access to the women's clinical notes and therefore could not ascertain the reason for consultation, the details of the clinician-patient discussion that took place or the outcome of the consultation. We are likely to have underascertained the number of comorbidities in nonconcession cardholders, as certain medications are below copayment. ${ }^{19}$ However, as the distribution of concession cardholders and number of Rx-Risk comorbidities were similar in both cases and controls, we do not expect any bias in the results. The sample for this study was drawn from the 45 and Up Study, limiting the sample to women aged $\geq 45$ years and consenting to linkage of their health records. Their health service history may differ from younger women or those who did not agree to participate in cohort studies. Given the small sample size, the results should be interpreted with caution. 


\section{Conclusions}

The evidence does not support the hypothesis that early endocrine therapy discontinuation is associated with recent clinical contact or investigations for cancer recurrence or metastasis. However, women who discontinued endocrine therapy were less likely to consult a clinician in the 6 months following discontinuation. Clinical contact cannot be assumed to support persistence with pharmacotherapy. Every consultation should be used as an opportunity to look into the women's breast cancer management. Of the clinician groups studied, general practitioners are best placed to engage and support women to continue pharmacotherapy. However, there needs to be mechanisms in place to prompt clinicians to do this at every visit and to contact women who have not had a recent consult.

\section{Acknowledgements}

This research was completed using data collected through the 45 and Up Study (www.saxinstitute.org.au/ our-work/45-up-study). The 45 and Up Study is managed by the Sax Institute in collaboration with major partner Cancer Council NSW and other partners: the National Heart Foundation of Australia (NSW Division), the NSW Ministry of Health, NSW Government Family \& Community Services - Carers, Ageing and Disability Inclusion, and the Australian Red Cross Blood Service. We thank the many thousands of people participating in the 45 and Up Study. The authors wish to thank staff at the Centre for Health Record Linkage, and data custodians the NSW Cancer Institute, the NSW Ministry of Health, the NSW Justice and Attorney General, the Australian Bureau of Statistics, and the Australian Government Department of Human Services. This study was jointly funded by Cancer Australia and the National Breast Cancer Foundation. FB was supported by the Friends of the Mater Foundation.

\section{Competing interests}

None declared

\section{Author contributions}

AKC, CS, FB, ER and DP conceived the study and interpreted data. DL performed the statistical analyses and drafted the manuscript. MB assisted with data interpretation and critically reviewed the statistical content of the manuscript. DP, CS, FB and MB assisted with acquisition of data and critically reviewed the manuscript. All authors read and approved the final manuscript and are accountable for all aspects of the work.

\section{References}

1. Cheung KL. Endocrine therapy for breast cancer: an overview. Breast. 2007;16(4):327-43.

2. Coates AS, Keshaviah A, Thurlimann B, Mouridsen $\mathrm{H}$, Mauriac L, Forbes JF, et al. Five years of letrozole compared with tamoxifen as initial adjuvant therapy for postmenopausal women with endocrine-responsive early breast cancer: update of study BIG 1-98. J Clin Oncol. 2007;25(5):486-92.

3. Arimidex, Tamoxifen, Alone or in Combination (ATAC) Trialists' Group, Forbes JF, Cuzick J, Buzdar A, Howell A, Tobias JS, Baum M. Effect of anastrozole and tamoxifen as adjuvant treatment for early-stage breast cancer: 100-month analysis of the ATAC trial. Lancet Oncol. 2008;9(1):45-53.

4. Davies C, Pan H, Godwin J, Gray R, Arriagada R, Raina V, et al. Long-term effects of continuing adjuvant tamoxifen to 10 years versus stopping at 5 years after diagnosis of oestrogen receptor-positive breast cancer: ATLAS, a randomised trial. Lancet. 2013;381(9869):805-16.

5. Bliss JM, Kilburn LS, Coleman RE, Forbes JF, Coates AS, Jones SE, et al. Disease-related outcomes with longterm follow-up: an updated analysis of the intergroup exemestane study. J Clin Oncol. 2012;30(7):709-17.

6. ATAC Trialists' Group. Results of the ATAC (Arimidex, Tamoxifen, Alone or in Combination) trial after completion of 5 years' adjuvant treatment for breast cancer. Lancet. 2005;365(9453):60-2.

7. van Herk-Sukel MP, van de Poll-Franse LV, Voogd AC, Nieuwenhuijzen GA, Coebergh JW, Herings RM. Half of breast cancer patients discontinue tamoxifen and any endocrine treatment before the end of the recommended treatment period of 5 years: a population-based analysis. Breast Cancer Res Treat. 2010;122(3):843-51.

8. McCowan C, Shearer J, Donnan PT, Dewar JA, Crilly M, Thompson AM, Fahey TP. Cohort study examining tamoxifen adherence and its relationship to mortality in women with breast cancer. $\mathrm{Br} \mathrm{J}$ Cancer. 2008;99(11):1763-8.

9. Hsieh KP, Chen LC, Cheung KL, Chang CS, Yang YH. Interruption and non-adherence to long-term adjuvant hormone therapy is associated with adverse survival outcome of breast cancer women-an Asian populationbased study. PLoS ONE. 2014;9(2):e87027.

10. Kemp A, Preen DB, Saunders C, Boyle F, Bulsara M, Malacova E, Roughead EE. Early discontinuation of endocrine therapy for breast cancer: who is at risk in clinical practice? Springerplus. 2014;3:282.

11. 45 and Up Study Collaborators, Banks E, Redman S, Jorm L, Armstrong B, Bauman A, et al. Cohort profile: the 45 and up study. Int J Epidemiol. 2008;37(5):941-7.

12. CHeReL: Centre for Health Record Linkage. Sydney: CHeReL; c2006 [cited 2015 May 14]. Available from: www.cherel.org.au 
13. CHeReL: Centre for Health Record Linkage. Sydney: CHeReL; c2006. Quality assurance; 2011 [cited 2015 Oct 16]; [about 3 screens]. Available from: www.cherel.org.au/ quality-assurance

14. Borgmeier I, Holman CD. Does vasectomy reversal protect against prostate cancer? Ann Epidemiol. 2004;14(10):748-9.

15. Brennan M, Butow P, Spillane AJ, Marven M, Boyle FM. Follow up after breast cancer - views of Australian women. Aust Fam Physician. 2011;40(5):311-6.

16. Hugo Centre for Migration and Population Research. Adelaide: University of Adelaide. ARIA (Accessibility/ Remoteness Index of Australia); 2015 [cited 2015 Jun 26]; [about 4 screens]. Available from: www.adelaide.edu.au/ apmrc/research/projects/category/about_aria.html

17. Vitry A, Wong SA, Roughead EE, Ramsay E, Barratt J. Validity of medication-based co-morbidity indices in the Australian elderly population. Aust N Z J Public Health. 2009;33(2):126-30.

18. Lu CY, Barratt J, Vitry A, Roughead E. Charlson and Rx-Risk comorbidity indices were predictive of mortality in the Australian health care setting. J Clin Epidemiol. 2011;64(2):223-8.

19. Paige E, Kemp-Casey A, Korda R, Banks E. Using Australian Pharmaceutical Benefits Scheme data for pharmacoepidemiological research: challenges and approaches. Public Health Res Pract. 2015;25(4):e2541546.

20. Zou G. A modified poisson regression approach to prospective studies with binary data. Am J Epidemiol. 2004;159(7):702-6.

21. Cummings P. Methods for estimating adjusted risk ratios. The Stata Journal. 2009;9(2):175-96.

22. Partridge AH, Wang PS, Winer EP, Avorn J. Nonadherence to adjuvant tamoxifen therapy in women with primary breast cancer. J Clin Oncol. 2003;21(4):602-6.
23. Owusu C, Buist DS, Field TS, Lash TL, Thwin SS, Geiger AM, et al. Predictors of tamoxifen discontinuation among older women with estrogen receptor-positive breast cancer. J Clin Oncol. 2008;26(4):549-55.

24. Huiart L, Dell'Aniello S, Suissa S. Use of tamoxifen and aromatase inhibitors in a large population-based cohort of women with breast cancer. $\mathrm{Br} \mathrm{J}$ Cancer. 2011;104(10):1558-63.

25. Hershman DL, Kushi LH, Shao T, Buono D, Kershenbaum A, Tsai WY, et al. Early discontinuation and nonadherence to adjuvant hormonal therapy in a cohort of 8,769 early-stage breast cancer patients. J Clin Oncol. 2010;28(27):4120-8.

26. Goss PE, Ingle JN, Pritchard KI, Muss H, Gralow J, Gelmon KA, et al. A randomized trial (MA.17R) of extending adjuvant letrozole for 5 years after completing an initial 5 years of aromatase inhibitor therapy alone or preceded by tamoxifen in postmenopausal women with early-stage breast cancer. J Clin Oncol. 2016;34(15 Suppl):LBA1.

27. Harrow A, Dryden R, McCowan C, Radley A, Parsons M, Thompson AM, Wells M. A hard pill to swallow: a qualitative study of women's experiences of adjuvant endocrine therapy for breast cancer. BMJ Open. 2014;4(6):e005285.

28. Kostev K, Waehlert L, Jockwig A, Jockwig B, Hadji P. Physicians' influence on breast cancer patient compliance. Ger Med Sci. 2014;12:Doc03.

29. Underhill C, Bartel R, Goldstein D, Snodgrass H, Begbie S, Yates $P$, et al. Mapping oncology services in regional and rural Australia. Aust J Rural Health. 2009;17(6):321-9.

30. Aiello Bowles EJ, Boudreau DM, Chubak J, Yu O, Fujii $\mathrm{M}$, Chestnut J, et al. Patient-reported discontinuation of endocrine therapy and related adverse effects among women with early-stage breast cancer. J Oncol Pract. 2012;8(6):e149-57.

\section{Copyright: (c) (7)(2)}

(C) 2017 Lopez et al. This article is licensed under the Creative Commons Attribution-NonCommercial-ShareAlike 4.0 International Licence, which allows others to redistribute, adapt and share this work non-commercially provided they attribute the work and any adapted version of it is distributed under the same Creative Commons licence terms. See: www.creativecommons.org/licenses/by-nc-sa/4.0/ 